\title{
Study and Evaluation of Innovative Fuel Handling Systems for Sodium-Cooled Fast Reactors: Fuel Handling Route Optimization
}

\author{
Franck Dechelette, Franck Morin, Guy Laffont, Gilles Rodriguez, Emmanuel Sanseigne, \\ Sébastien Christin, Xavier Mognot, and Aurélien Morcillo \\ Commissariat à l'Energie Atomique et aux Energies Alternative (CEA), Centre de Cadarache, 13108 Saint Paul-Lez-Durance, France \\ Correspondence should be addressed to Franck Dechelette; franck.dechelette@cea.fr
}

Received 19 June 2013; Accepted 3 December 2013; Published 11 February 2014

Academic Editor: Gianfranco Caruso

Copyright (C) 2014 Franck Dechelette et al. This is an open access article distributed under the Creative Commons Attribution License, which permits unrestricted use, distribution, and reproduction in any medium, provided the original work is properly cited.

\begin{abstract}
The research for technological improvement and innovation in sodium-cooled fast reactor is a matter of concern in fuel handling systems in a view to perform a better load factor of the reactor thanks to a quicker fuelling/defueling process. An optimized fuel handling route will also limit its investment cost. In that field, CEA has engaged some innovation study either of complete FHR or on the optimization of some specific components. This paper presents the study of three SFR fuel handling route fully described and compared to a reference FHR option. In those three FHR, two use a gas corridor to transfer spent and fresh fuel assembly and the third uses two casks with a sodium pot to evacuate and load an assembly in parallel. All of them are designed for the ASTRID reactor (1500 MWth) but can be extrapolated to power reactors and are compatible with the mutualisation of one FHS coupled with two reactors. These three concepts are then intercompared and evaluated with the reference FHR according to four criteria: performances, risk assessment, investment cost, and qualification time. This analysis reveals that the "mixed way" FHR presents interesting solutions mainly in terms of design simplicity and time reduction. Therefore its study will be pursued for ASTRID as an alternative option.
\end{abstract}

\section{Introduction}

In the framework of the French Act of June 28, 2006, about nuclear materials and waste management, a Generation IV and actinides incineration demonstration prototype is to be commissioned in the 2020 decade [1]. This prototype called ASTRID (Advanced Sodium Technological Reactor for Industrial Demonstration) sets out to demonstrate advances on an industrial scale by testing innovative options in areas earmarked for improvement (in particular safety, operability, and inspection and repair). R\&D program led currently in support to the selection of ASTRID options, particularly in the following topics:

(i) core design with the objective of reducing the probability of core meltdown and/or limiting the energy release during potential accidents, development of innovative third shutdown system, and improvement in core monitoring;

(ii) improvement of decay heat removal (DHR) systems performances, with the development of an efficient system through the reactor vessel and the integration of DHR heat exchangers in intermediate heat exchangers;

(iii) development of a strategy in support of the limitation of core melting consequences including the R\&D in support to the development of the core catcher;

(iv) development of innovative heat exchangers for a gasbased energy conversion systems (ECS) as an alternative to the classical steam cycle;

(v) development of innovative fuel handling systems (FHS). 
In terms of economy, Generation IV systems shall be competitive, for the same overall performance, compared to other sources of energy at the time they will be put into operation $[2,3]$. This means a lot of efforts with regard not only to investment costs but also to availability and operation costs. These requirements impact fuel handling systems such as the following.

(i) After a learning period, the reactor must demonstrate a high load factor (e.g., up to 90\%).

(ii) The investment cost of the prototype shall be minimised, with technical options compatible with future commercial reactors deployment. This option is particularly relevant in FHS selection which can influence several parts of the reactor block design: from the primary vessel diameter until the balance of plant and plant layout. The ratio of FHS (including external vessel storage tank (EVST), Casks, and civil engineering) in the total reactor investment cost is estimated from $15 \%$ to $20 \%$.

From 2007 to 2009, R\&D investigations in FHS aim to review design options $[4,5]$, experimental feedback from previous French sodium-cooled fast reactors (SFR) and international reactors [6-9], and to cross this review analysis with recent innovative options proposed by the scientific community [10-12]. This work leads to a first set of innovative preselected options [13] and to determining several axes of R\&D development to pursue for ASTRID design options. In the field of SFR fuel handling innovation, this paper aims to describe studies carried on since 2011 by CEA and in cooperation with COMEX Nucléaire and Bertin/CNIM as mechanical systems designers, and to characterise options regarding ASTRID criteria.

\section{Scope of Work}

Before defining the several routes chosen in the past and that could be investigated for the future, a review of the different options has been carried out using the fast reactor database and recent technological development in SFR design. The considered options concern fuel handling systems (under rotating plugs), transfer assemblies options between reactor vessel and external storage, and also, in the particular case of fuel handling through gas corridor, fuel handling in the EVST. The work performed is a characterisation of solutions, a performance review, and an analysis of the main advantages and drawbacks of the options compared to a so-called Starting Reference Solution (SRS) based upon well-known French SFR options or some option already envisaged in French project, that is, EFR reactor [14]. The main features of the SRS are described below.

(i) The primary in-vessel FHS is composed of two rotating plugs (Superphenix and EFR option).

(ii) A direct lift charge machine is placed in the centre of the Above Core Structure (ACS) (EFR option). It is used for removal and insertion of core components belonging to the inner handling zone. (iii) A fixed arm charge machine is placed on the large rotating plug (Phenix and EFR option). It is used for removal and insertion of core assemblies belonging to the outer handling zone. Furthermore, it forms the link between the load-unload station in the reactor and the direct lift charge machine using intermediate put-down/take-over positions at the inner core zone boundary.

(iv) The load-unload station in the reactor is an equipment supported by the reactor (Phenix and Superphenix option).

(v) The fuel assembly evacuation is performed using a sodium pot for its permanent cooling (Phenix and Superphenix options).

(vi) A fuel handling cask leads to evacuate fuel assembly from the primary vessel to the in-sodium external vessel storage tank (Rapsodie and EFR option, but, was designed with no sodium pot in both cases, only gas cooling system and with low residual power spent fuel).

(vii) There is no rotor system (exchange new/spent fuel assembly), neither in the reactor vessel nor in the external storage.

The SRS option is represented in Figure 1.

\section{List of the Main Innovation Selected}

Starting from the SRS route, several innovations were selected either on some specific and targeted study on a single component or on a global approach on fuel handling route (FHR) from the primary vessel to the EVST. In a first step, a large survey has been performed on innovative ideas without constraints or restrictions regarding maturity and cost level. Then, technological feasibility conclusion study is presented for each option, and a criteria grid analysis has been performed to highlight innovative options to persue for ASTRID. The following options have been investigated concerning the single route optimization:

(i) Above Core Structure (ACS) designed in two parts (one in the small rotating plug, SRP, and the other in the large rotating plug, LRP),

(ii) Pantograph Arm Machine associated with a slit ACS,

(iii) design of the "Dual Location Rotor."

(iv) design of the "Simultaneous Handling of two fuel assemblies."

A second review [15] has been carried out and concerns specific component optimization (SCO).

\section{Fuel Handling Route Optimization: Description of the "Mixed Way" Option (Ramp and Transfer Lock, Gas Corridor)}

4.1. Motivations. This option is investigated in order to mutualize the necessary equipment to twin nuclear plant 


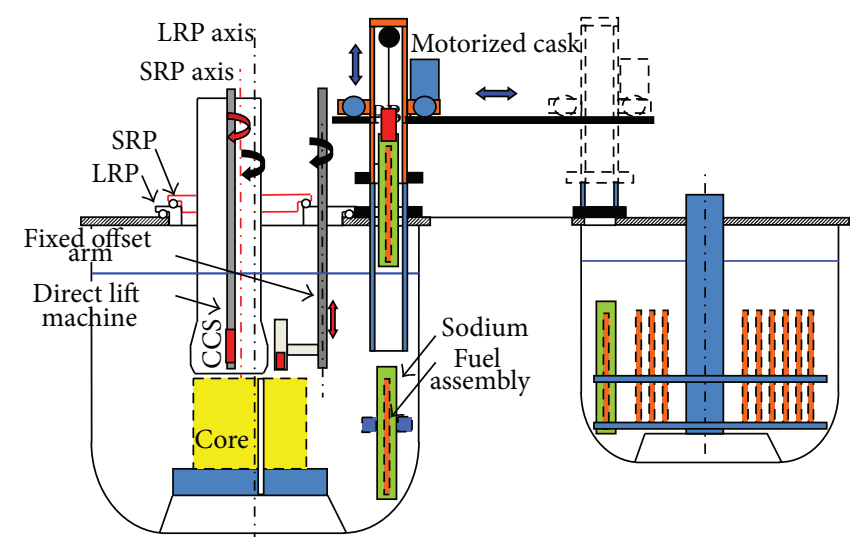

FIGURE 1: View of the fuel handling route called SRS.

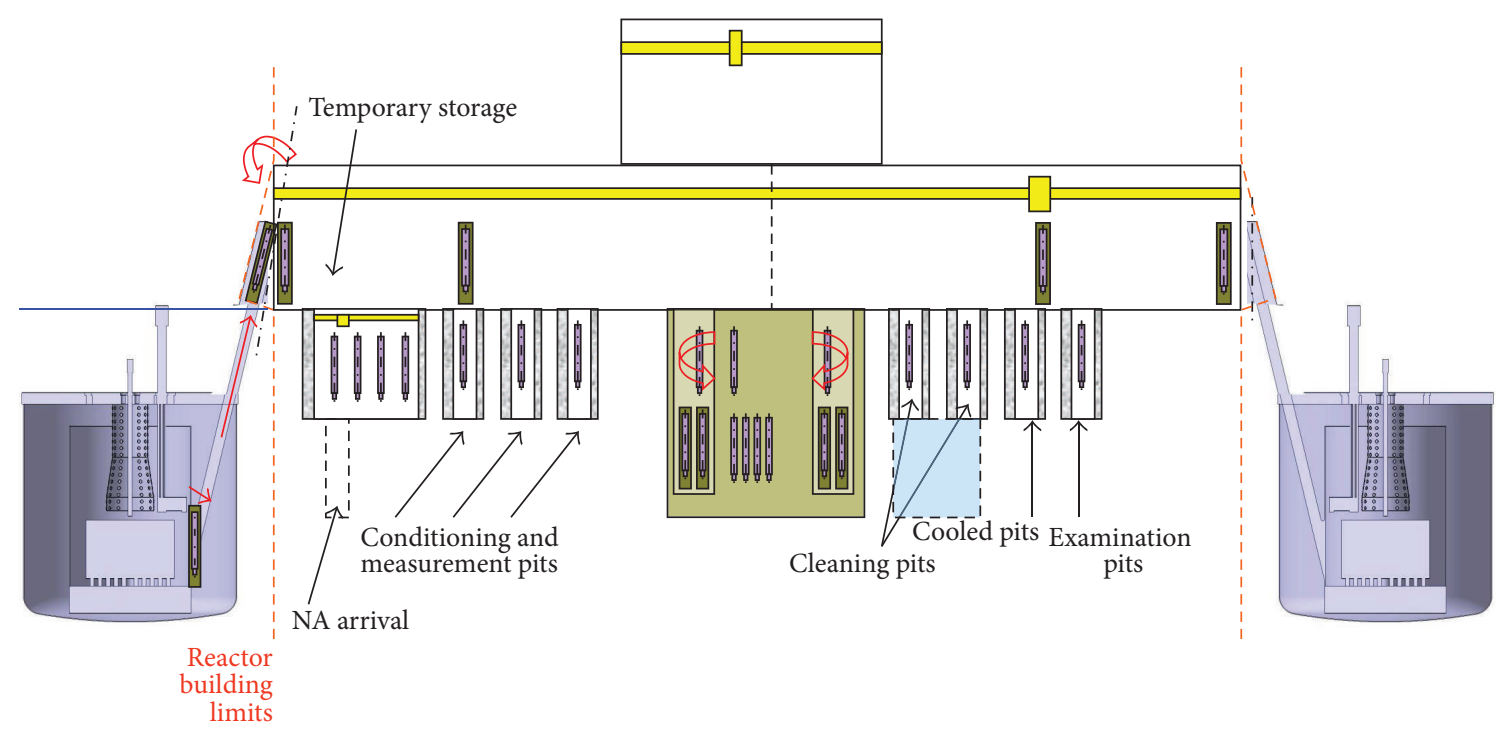

FIGURE 2: View of the fuel handling route shared by two nuclear vessels.

units, for the development of an industrial fleet of commercial SFR [16]; see Figure 2. The standard transfer lock/charge and discharge ramp fuel assembly transfer system deployed on Phenix and Superphenix reactors can be used in a singleunit facility but cannot be integrally applied with a twinunit option as the external storage unit would be too close to each reactor vessel. Indeed, distance between reactor buildings would not be compatible with facility safety and operability requirements. Therefore a "mixed way" including a transfer lock/ramp charge and discharge associated to a gas corridor has been developed to overcome the problem of reactor building proximity, with optimized simplification of elementary operations, robustness, and availability.

The transfer lock/charge and discharge ramp solution, which is reliable and for which there is a significant feedback [7], presents the advantage of avoiding the displacement of a very massive cask inside and outside the reactor containment.
However, it is necessary to study the possible difficulties and key points raised by using a gas corridor mainly in terms of gas volume management and interaction with sodium, thermal behaviour of the assembly handled in this corridor during normal or degraded situation, and management of sodium drips along the fuel handling path.

4.2. Technical Characteristics. Technological solutions characteristics constituting this mixed way fuel handling route are described below.

Ramp and Fuel Transfer Pot in the Reactor Vessel. The solution features no real innovation in relation to the one developed for Phenix, mainly due to internal vessel geometry, that is, the implementation of a conical inner vessel. The angle of slope of the ramp is also standard, that is, around $17^{\circ}$. The fastening of the fuel pot and its tilting feature are however innovative as 


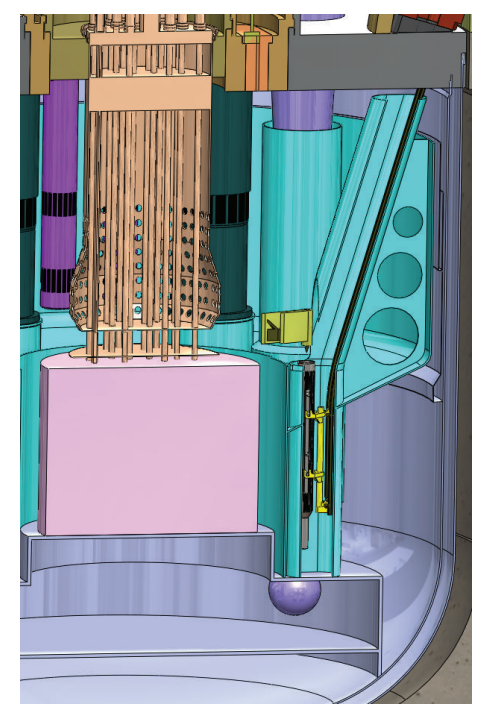

FIGURE 3: Sodium progressive tilting proposed.

the fuel pot needs to be transported from the primary vessel to the external storage unit with a separation of the casks. The fuel transfer pot has been designed to progressively tilt the pot using the specific curvature of the rail supporting the pot (see Figure 3). The fuel transfer pot can be lifted up and down using a winch and a chain at the top of the rotating transfer lock.

The fuel transfer pot moving along the ramp comprises the following main components described in Figure 4.

Interface Rotating Transfer Lock. The fuel handling exit transfer lock is located on the reactor roof, supported by the concrete floor with a valve to ensure isolation from the primary circuit at the argon cover gas plenum. This rotating transfer lock comprises a stainless steel envelope preventing the air from entering the fuel handling transfer lock (argon overpressure), as well as an external biological shielding (steel and lead) with a $500 \mathrm{~mm}$ thickness. The transfer lock itself slopes in relation to the floor by the half-angle of the ramp, that is, $8.5^{\circ}$, to present the sodium pot-holding truck directly in a vertical position for transfer into the corridor. Each opposite side of the rotating part comprises two translation rails for the pot following on from the ramp as well as an associated secured hoist (emergency brake and additional descent device) and a chain reel. The rotating part has an offset motor for access outside the biological protection and argon environment. The gas corridor provides the link to the fuel handling building. Figure 5 describes the main components of this rotating transfer lock.

Fuel Handling Gas Corridor. The gas corridor and its interface with the rotating transfer lock comprise an internal metal envelope (liner) ensuring corridor tightness and concrete biological protection (1 meter) to maintain a green zone outside in all circumstances. All the motors and actuators are placed outside the gas corridor. A camera viewing and image transport system enables the fuel handling process

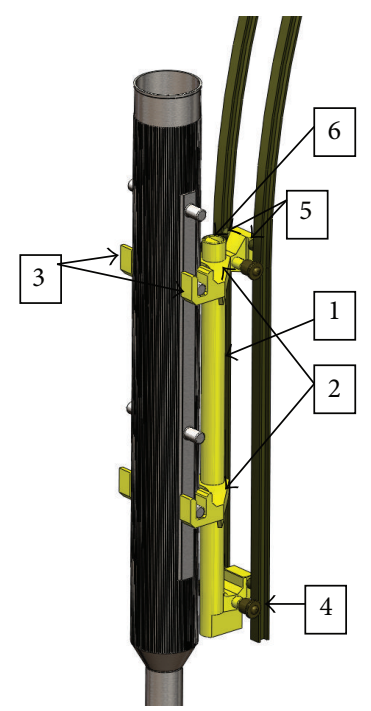

FIGURE 4: Intravessel fuel transfer pot and ramp (1: massive common section, 2: gripper cradle, 3: embedded handles, 4: translation wheel, 5: holding counterwheel, and 6: chain fastening).

to be monitored. The ambient temperature and pressure levels are also monitored. A drainer collecting sodium drip is located at the corridor floor. A general ventilation system for the corridor evacuates the heat from the handled sodium pot. Two storage pits with active cooling system are set in the path of the gas corridor, between the positions of the reactor primary vessel on one side and of the EVST on the other. These pits are used to return to a secure situation for cooling, provided that the residual power extraction system is sufficiently efficient and passive for a period given.

The pot translation with horizontal transfer mechanisms takes place using a cable/wheel system driven by two synchronized motors, placed outside. Above the access to the external storage unit, a hoist lifts up and down the pot holder to set down and pick up the sodium pot in the external storage vessel.

An isolation valve is located in the lower part of the access door to the external storage pit, and another one is located on the upper roof of the EVST. The pot-holder truck accessing the EVST moves along vertical rails to a low position in the vessel, to handle the assembly using the grabbing arm coupled to its rotating plug. With the geometry described in this study, it is possible to store approximately 340 assemblies for a main vessel diameter of 6.3 meters. The extrapolation to a twin commercial reactor can be obtained by symmetry of the gas corridor, since the pot transfer between the gas corridor horizontal transfer mechanism and the lift truck of the external storage unit can take place with arrival from the left or from the right.

Figure 6 shows a design of the mixed way FHR. The distance between the two axes of the reactor vessel and the EVST is approximately $23 \mathrm{~m}$.

Primary Vessel Fuel Handling and Transfer. The solution selected is based upon two rotating plugs, a takeover position, 


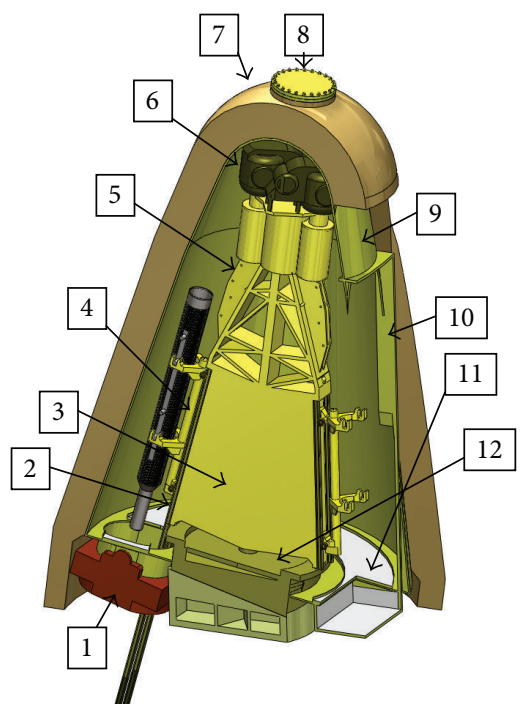

(a)

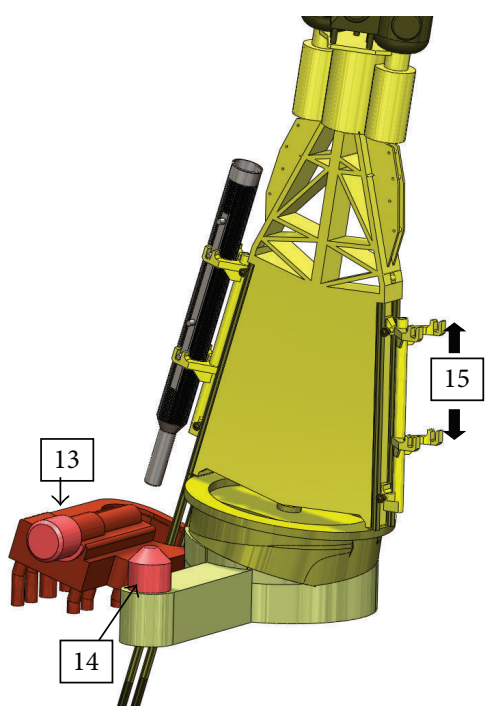

(b)

FIGURE 5: Detailed view of the ramp/gas corridor interface rotating part (1: primary circuit isolation valve, 2: ramp continuity rail, 3 : inclined rotating part, 4: pot-holding truck in upper position, 5: chain guide and reel, 6: sealed secured hoist 7: biological shielding, 8: tight passage and motor access, 9: metallic envelope, 10: gas corridor link/exit interface, 11: dripping path and sodium recovery, 12: rotating part base, 13: isolation valve motorization unit, 14: rotating part motorization and drive unit, and 15: set-down and pickup of the sodium pot).

and a fixed gripper arm on the large rotating plug (Figure 6). The angle of the ramp is around $17^{\circ}$.

Kinematic Transfer Applied to the Evacuation of an Irradiated Fuel Assembly. The fuel assembly discharge kinematic can be described in 9 major steps as presented in Figure 7.

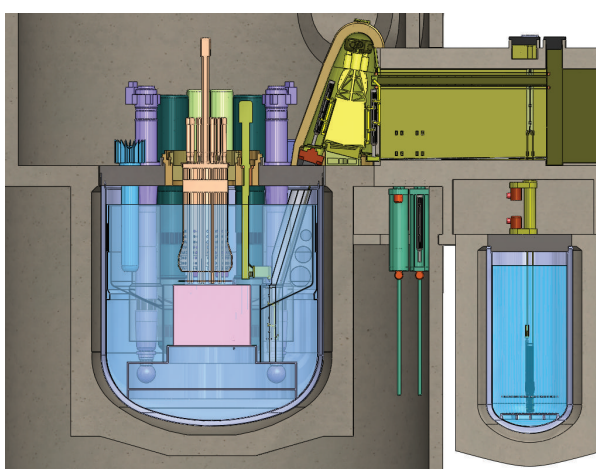

Figure 6: Vertical cross-section of the mixed way fuel handling route.

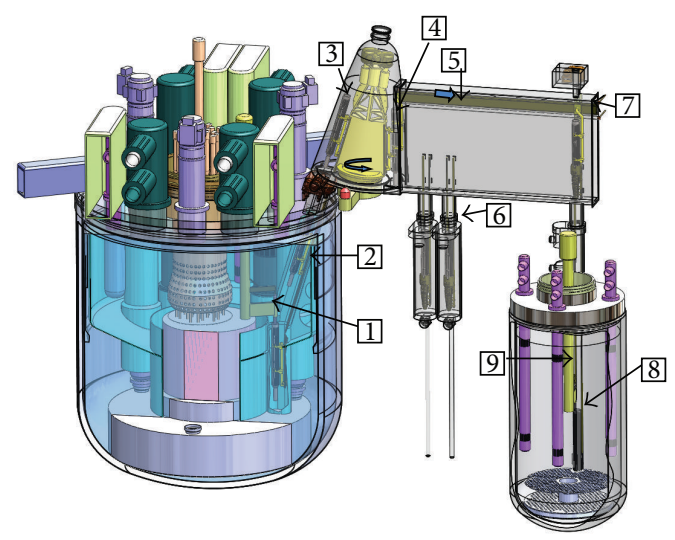

FIgURE 7: Kinematics for the evacuation of an irradiated assembly in sodium pot (1: assembly set-down in the pot, 2: pot moving up the ramp, 3: arrival at the upper stop of the rotating transfer lock, 4: rotation of the transfer lock in position, arrival of the corridor transfer mechanism in position, truck moving down the ramp, and pot set-down, 5: translation of the corridor transfer mechanism, 6: docking of pot holder associated with the temporary cooling pit and descent in the pit, 7: arrival in the EVST + pot lifting using the pot holder up to detachment and disconnection, 8: pot-holder descent to the lower position of EVST, and 9: assembly fuel handling and set-down in storage unit using the fixed arm).

The cooling of the sodium pot containing a fuel assembly in the gas corridor (from the primary sodium exit to the entry into the EVST) must be studied according to the external design of the pot (pot designed to enhance heat convection with fins and forced ventilation of the transfer lock and corridor). The failure and blockage modes during displacements must also be investigated, especially with return to a safe cooling state to be ensured in any configuration (in primary vessel, in external storage unit, or in temporary storage pit with passive cooling system), and fast enough to avoid fuel failure. Depending on the time to return to safe position, a residual power value per fuel assembly authorised for evacuation may be determined. The blockage risk of the transfer mechanism during translation in the corridor, loss of 
power or drive motor failure, and the breaking of drive cables of the transfer mechanism must be studied and remedied using backed-up systems to return to a safe cooling position for the pot. A fuel handling rate may also be defined using this kinematic chain, according to the values usually taken for truck and horizontal transfer mechanism movement speeds, valve opening, pot dripping time, and so forth.

Internal Fuel Handling in the EVST. The solution reuses standard primary fuel handling elements, that is, a central rotating plug with an integrated fixed offset arm. The overall height of the arm, with its mechanism, is limited to allow access to the lower part of the corridor. The design of the external storage unit takes into account the location of decay heat removal exchangers and the number and the location of storage positions (340 locations in this case). Residual power in the EVST is evacuated through three sodium/sodium heat exchangers placed at $90^{\circ}$ around the vessel, accessible by a standard crane in the upper part of the building.

4.3. Technical Data for Option Qualification. The main new points to be qualified for this option are the pot thermal hydraulic with the hottest assembly in sodium; the management of sodium drips and aerosols in the inert atmosphere of the gas corridor and the rotating transfer lock; hoist mechanisms embedded in the rotating transfer lock; and demonstration of return to a safe state in case of transfer mechanism movement failure.

\subsection{Option Performance Review}

Economic Review. As a first rough estimation, this solution seems less costly than the one corresponding to the SRS version, due to the absence of wide reactor containment opening and of a heavy cask transfer.

Safety Approach Review. As a first study, this solution seems favourable with regard to earthquakes than a heavy cask circulating close to the reactor slab. The third containment barrier seems easier to maintain using a set of isolation valves rather than a wide opening of the reactor containment. As regards cooling the sodium pot containing the assembly being handled, the demonstration is more complex to realize than the cask, although it does not lead to a technical impossibility. But alternatively this analysis could lead to a decrease of the maximum residual power admitted for fuel handling which would impact the defueling strategy and consequently the load factor. Here relies the key point of this option.

ISIR Review. The instrumentation and monitoring of temperature parameters of the handled pot are slightly trickier to manage in a gas corridor than in a cask.

Operability Review. The ramp and transfer lock solution has been operational in Phenix and in Superphenix reactors. Furthermore, this fuel handling system using the gas corridor has already been implemented and operated on the UK prototype fast reactor [17-20]. Feedback is therefore significant and good operability is estimated. Feedback handling time is optimized by minimizing and simplifying the movements and transfers of the entire kinematics chain, except for the takeover position. A short fuel handling transfer time might therefore be obtained, if a correct and continuous cooling of the assembly can be obtained.

Design Complexity Review. The ramp and transfer lock solution is industrially known and its implementation is not very sophisticated. The insertion of the gas corridor, the management of its inert atmosphere, and the global kinematic chain make the design more complex compared to a caskbased displacement solution, but it does not pose any significant problems or incur any major extra costs in relation to the dimensioning of a reactor containment integrating a large opening for the cask transfer. The design complexity is therefore considered as medium compared to a cask-based solution.

Extrapolation to a Power Reactor. The solution can be twinned with a second nuclear island as previously explained.

\section{Fuel Handling Route Optimization: Description of the "Three Rotating Plugs, Ramp and Gas Corridor" Option}

5.1. Motivations. This concept is slightly similar to the previous one. It has been studied in collaboration with Cea and Comex Nucléaire engineering nuclear company. This alternative solution presents potential advantages: fuel handling was possible without takeover position in the primary vessel: no fuel handling arm, only vertical lifts; minimisation of mechanisms in the vessel, two direct lift machines make fuel handling more flexible and restrict rotating plugs movements; no fastening and unfastening pot requirements because of a single transfer system use for both operations.

5.2. Technical Description of the Solution. The elements forming this fuel handling option are shown in Figure 8. The steps for handling an irradiated assembly are as follows.

Fuel Handling in the Reactor Vessel. The system allows the assemblies to be transferred between the core and the horizontal transfer mechanism pot (HTMP). This equipment comprises the following subsystems: three rotating plugs and two direct lift machines. The rotating plugs can place the direct lift machines above any assembly of the core and above the horizontal transfer mechanism pot. The direct lift machines are used to lift an assembly up and down either in the core or in the horizontal transfer mechanism pot. Each direct lift machine is fitted with a grabbing system locking an assembly during fuel handling. This system also provides an 


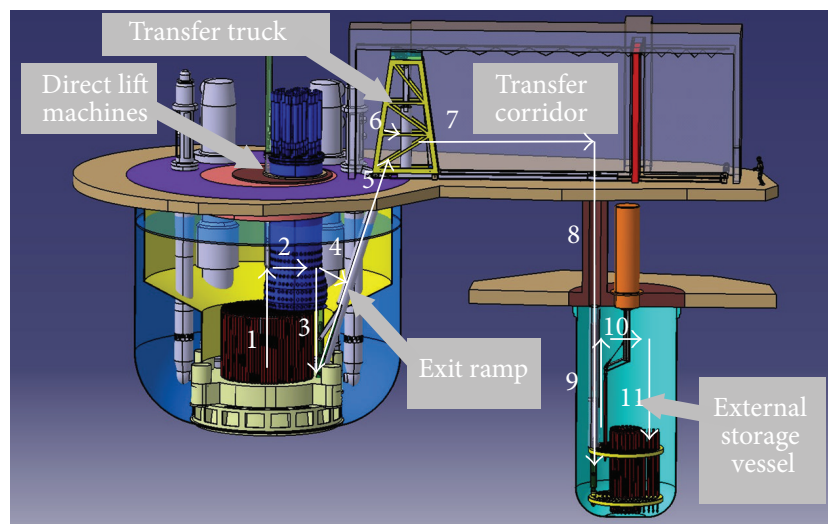

FIGURE 8: Steps of the three-rotating-plug solution in detail (1: the assembly is extracted from the core using one of the two direct lift machines, 2: the rotating plug movements position the direct lift machine above the horizontal transfer mechanism pot (HTMP), 3: the assembly is lifted down into the HTMP, 4: the HTMP is tilted in the exit ramp, 5: the HTMP is driven back up the ramp to its position in the transfer truck, 6: the HTMP is tipped vertically into the transfer truck, 7: the transfer truck moves along the corridor to its position above the external storage vessel, 8: the HTMP is lifted down vertically into the EVST, 9: the assembly is picked up by a fuel handling arm and removed from the HTMP, 10: the fuel handling arm positions the assembly above its storage location, and 11: the fuel handling arm sets the assembly down in its location).

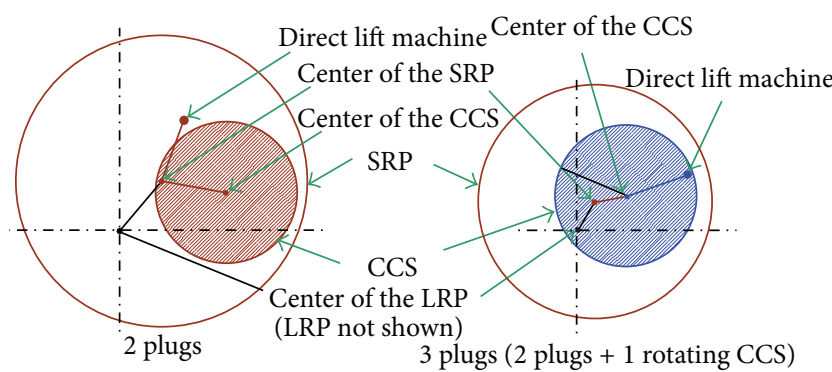

FIGURE 9: Two plugs/three plugs kinematics comparison.

angular orientation of the assemblies being handled (rotation about a vertical axis).

The Number of Rotating Plugs. Two rotating plugs are sufficient to reach any location in the core with direct lift machines. Using a rotary ACS supporting the direct lift machines limits the centre-to-centre distance between the large rotating plug and the small rotating plug, and consequently reduces the SRP size (Figure 9). In order to reduce the diameter of the primary vessel, a third rotating plug was added.

Direct Lift Machines. Direct lift machines are used to raise and lower the assemblies (Figure 10). There are two rods to

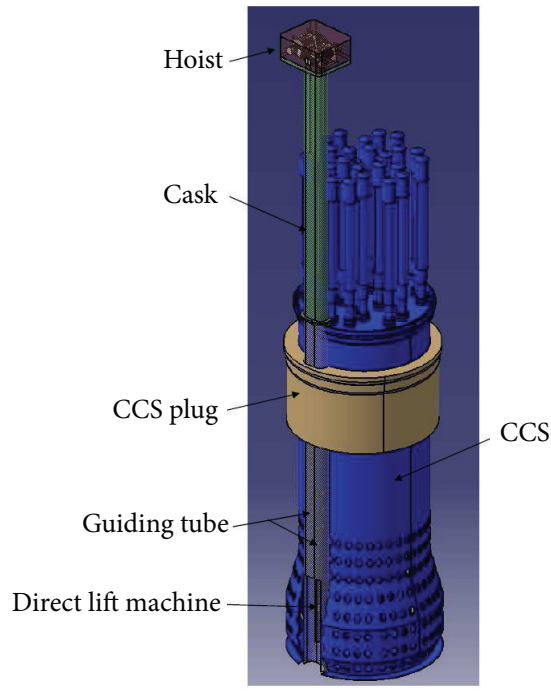

Figure 10: Detail of the fitted direct lift machines.

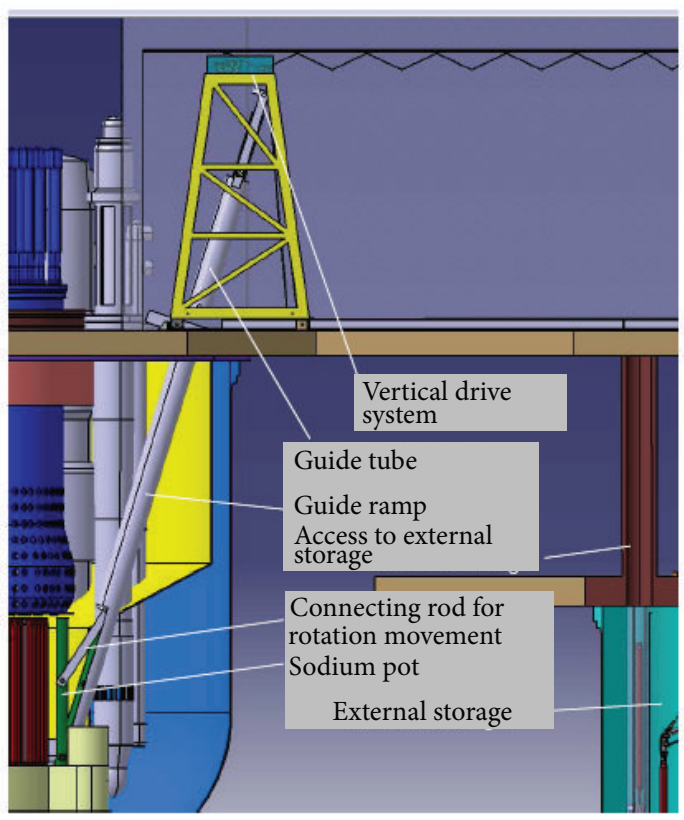

FIGURE 11: Horizontal transfer mechanism pot transfer system.

carry out operations in parallel inside the vessel. When the ACS comes into position above the sodium pot to set an irradiated assembly down, the sodium pot is loaded with a new assembly. The second direct lift machine is then used to extract the new from the sodium pot, to place the irradiated assembly carried by the first direct lift machine.

Subassembly Transfer System. The horizontal transfer mechanism (Figure 11) performs transfers between the reactor vessel (loading/unloading position) to the EVST. This 


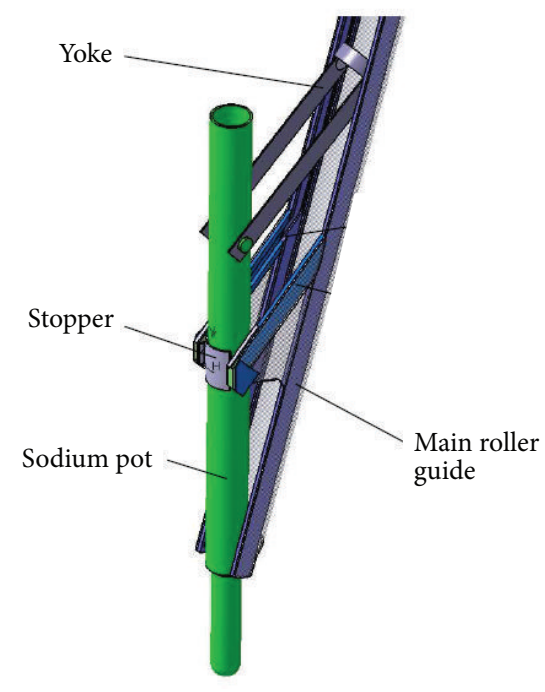

Figure 12: View of the ramp system.

system comprises three sub-systems: equipment related to the reactor vessel (the extraction ramp and the reactor vessel shutoff valve); the equipment related to the transfer truck (the horizontal transfer mechanism pot, the pot descent system) and its equipped truck; the equipment related to the EVST (support of the pot in the storage vessel, vertical ramp, and storage vessel shutoff valve).

Reactor Vessel Entry/Exit System. This system comprises the equipment related to the reactor vessel: the shutoff valve for the opening of the reactor slab, the horizontal transfer mechanism for pot guiding, and straightening ramp. A stop piece is positioned at the end of this ramp to keep the HTMP vertical (Figure 12).

Transfer Subsystem. This sub-system comprises the HTMP with its shell and the manoeuvring systems for raising/lowering, the mechanically welded structure forming the truck and the translation systems, the guiding tube housing the horizontal transfer mechanism pot and orientating it for introduction into the vessel, and the transfer corridor.

The transfer corridor is fitted with two rails guiding the truck and sealed penetrations for manual intervention in the event of an incident on the drive system units positioned above the truck. The thickness of the corridor protects operators against radiation. The assemblies are cooled down by argon at a maximum temperature of $50^{\circ} \mathrm{C}$ in the transfer corridor.

5.3. Technical Data for Option Qualification. The main new points to be qualified for this option are as follows: thermal hydraulic features of the pot (in sodium at handling temperature, irradiated, and new assembly), management of drips and behaviour in sodium aerosol atmosphere (mockup representative of part of the corridor, outside biological protection), and failure modes for the horizontal transfer mechanism displacement.

\subsection{Option Performance Review}

Economic Review. Compared to the SRS, it appears that fuel handling in the vessel is slightly more expensive due to the addition of the third plug and the minimal increase in the vessel diameter. In addition, the cost of a transfer corridor is to be compared to the cost of a cask and the cost of a reactor building extension during fuel handling, making the corridor solution less costly at first approach. The gas corridor has significant larger volume than in the mixed way solution, inducing cost increase in terms of argon gas purification, temperature, and pressure control equipment. Nevertheless this solution is considered in its overall to be less costly than the reference solution.

Safety Review. As regards safety, this solution seems equivalent to the "mixed way" solution.

ISIR Review. The number of items of equipment in the vessel is equivalent. However, inspection and repair in the transfer corridor may be an issue; a negative point is counted.

Operability Review. On the whole, this solution is equivalent to the mixed solution or slightly better thanks to the removal of the takeover position in the vessel.

Design Complexity Review. The major difference lies between the cask and the transfer corridor. The management of fluids, power, and information feedback is made more complex by adding a third rotating plug.

Extrapolation to a Power Reactor. This solution can be extrapolated to a future commercial reactor.

\section{Description of the "Cask and Direct Fuel Handling" Route}

6.1. Motivations. This option has been studied in collaboration with Bertin/CNIM mechanical systems designer company. This preliminary design presents the following advantages: no mechanism in the vessel during the reactor operation, optimized cooling of the assembly during the transfer using the cask (through integrated active cooling systems), and the transfer lock is ensuring continuity of the confinement of the reactor building. This solution can be considered as an innovative evolution of the SRS option.

6.2. Technical Description of the Solution. The equipment required for the primary fuel handling is described as Figure 13: an LRP installed on the slab of the reactor vessel, an SRP installed on the LRP, a sealed penetration installed on the SRP fitted with a shielded valve sealing the penetration during fuel handling campaigns, a direct lift machine centred on the ACS, two fuel handling casks, roller rails for cask transfer, a transfer lock between the fuel handling building and 


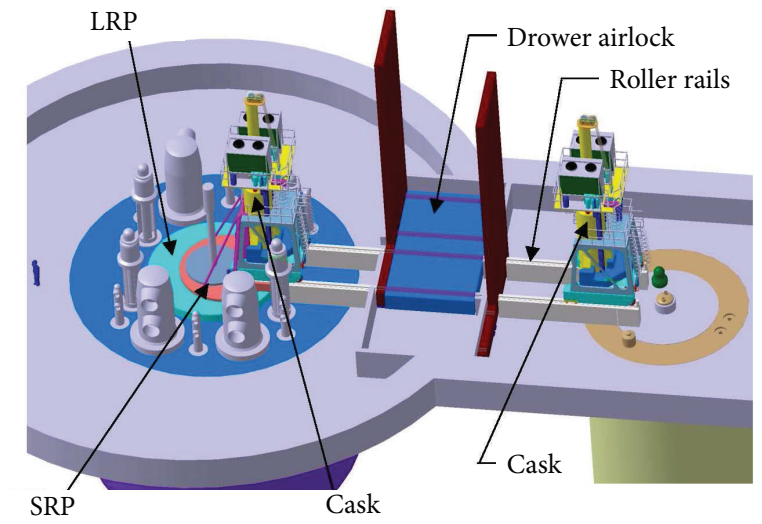

FIGURE 13: Main components of the Bertin/CNIM fuel handling route.

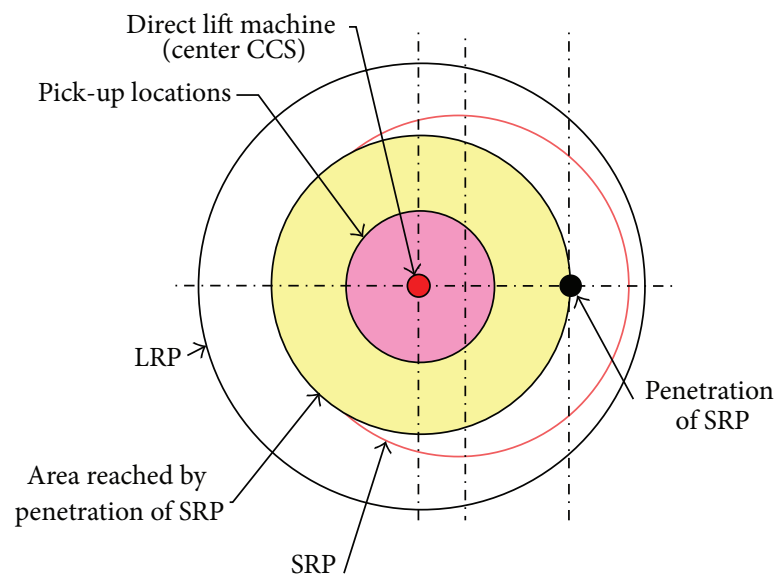

FIGURE 14: Retention of the direct lift machine at the centre of the ACS.

the reactor building, a rotating plug on the slab of the EVST, a fuel handling arm installed on the rotating plug of the storage vessel, and a pick-up station integrated into the EVST.

Fuel Handling in the Vessel. The combined rotations of the two rotating plugs provide access to all the core fuel assemblies. To minimise the impact on the diameter of the primary vessel, the direct lift machine installed at the centre of the ACS is kept (Figure 14).

Structures Involved in Cask Holding and Displacement. The rolling rails allow the truck to take position on the SRP. They are in two parts: a first fixed part on the reactor slab and a second mobile part linked to the SRP. Accordingly, these rails will follow the SRP when rotating. Two electrically controlled screw jacks will support the rails when the truck passes (Figure 15).

A cask support structure, integral with the SRP, locks the cask when the truck is removed. The cask/structure link is ensured by two locking hooks and holds the cask in the event of an earthquake.

Transfer Cask. It is used to extract a spent fuel element from the reactor vessel, transfer it and place it in the EVST, and extract a new fuel element from the EVST. The cask comprises the following subsystems: a cask casing, a sodium pot (where irradiated assembly is cooled), a fuel grabbing tube, and a cask cooling system (Figure 16).

The lower part of the pot comprises a plug valve sealing the pot. This valve is controlled by a rack located at the pot guiding tube. When the pot descends into the vessel, the toothed shaft of the valve engages in the rack and rotates the pot. The bearings of the journals of the plug valve are specially designed to be disengageable from the top of the pot to open the valve even in the event of seizure (Figure 17).

In this cask the spent fuel must be continuously cooled. The cooling system architecture is done by argon flowing in a circuit in the cask and cooled down by the units outside the cask by blowers (set with two redundant cooling units). The cooling units are installed on a floor integrated into the cask with mechanical uncoupling.

Airlock. There is an airlock between the reactor building and the fuel handling building to transfer simultaneously two casks through a fuel handling path (Figure 18). The airlock comprises two doors with inflatable seals. In closed or open position, the doors are automatically locked by motors. A control unit balances the pressures in the airlock when opening towards one of the buildings. The fuel handling path is installed inside the airlock. It features two running rail locations, the first one to support a cask and the second one to allow the cask to go from one building to the other.

6.3. Technical Data for Option Qualification. The two main points to qualify are

(i) the cask featuring direct extraction with its sodium pot with opening in the lower part:

(a) the plug valve qualification: operation in air, sodium, and temperature environments, behaviour from aerosol deposits, ageing of the bearings and the seals, remote controller, and degraded mode operation,

(b) the guiding tube system qualification with integrated grab,

(c) the truck behaviour when loaded,

(d) the isolation valves of the reactor vessel qualification (in sodium aerosols);

(ii) the reactor building qualification exit airlock.

\subsection{Option Performance Review}

Economic Review. This option is considered more costly than the SRS solution, mainly due to the need for two casks working in parallel to obtain a correct fuel handling time, the 

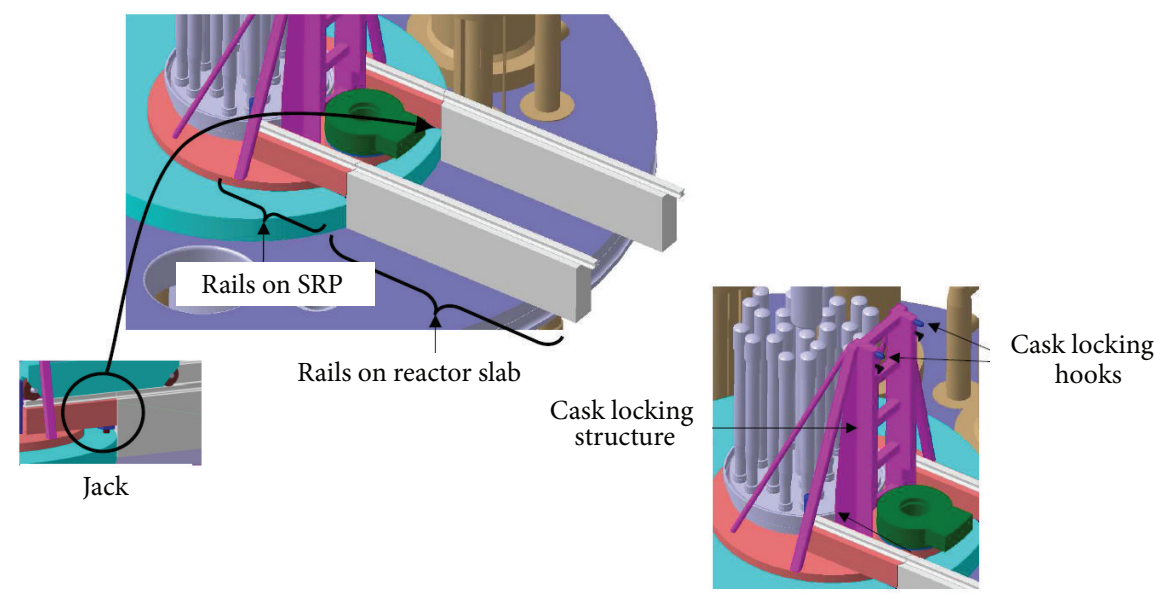

FIGURE 15: Details of the running rails and cask holding structure.
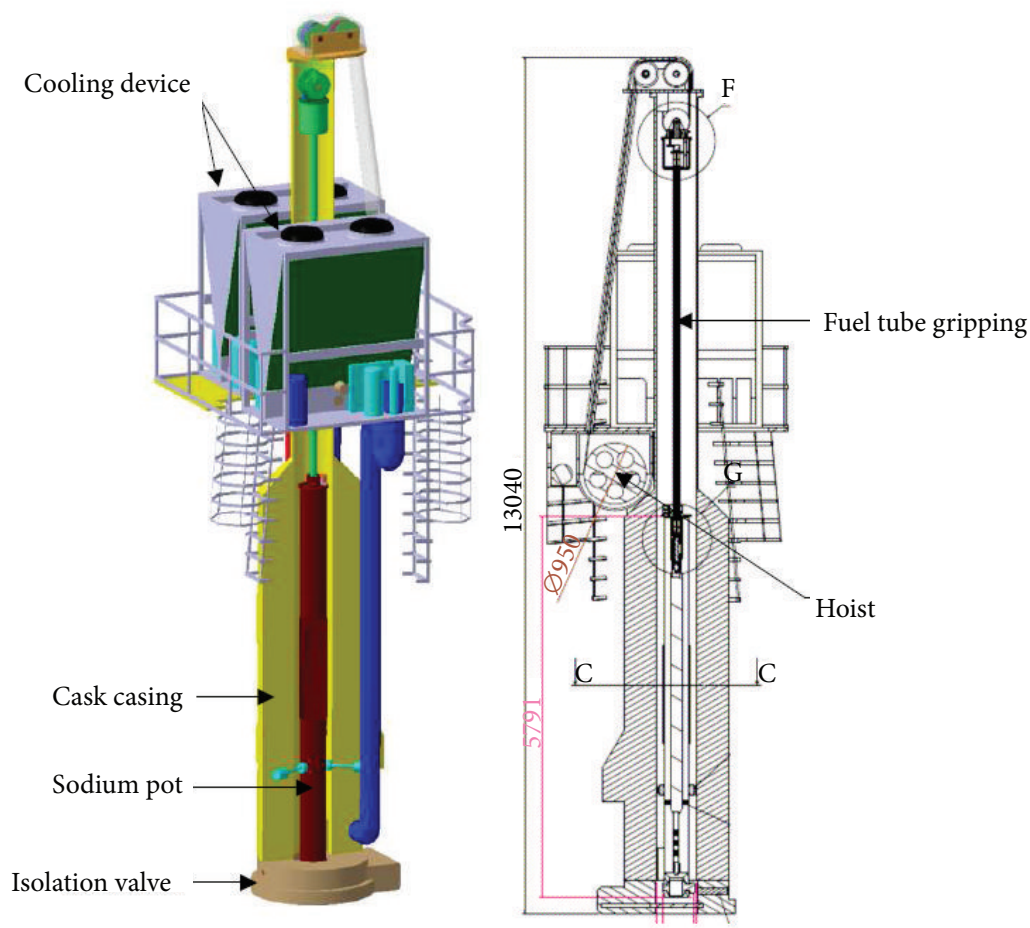

FIGURE 16: Overview of the transfer cask.

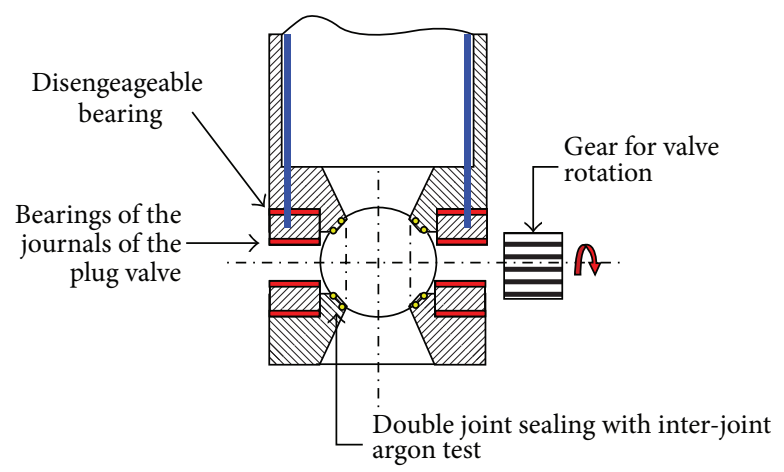

FIGURE 17: Plug valve at the bottom of the pot. 


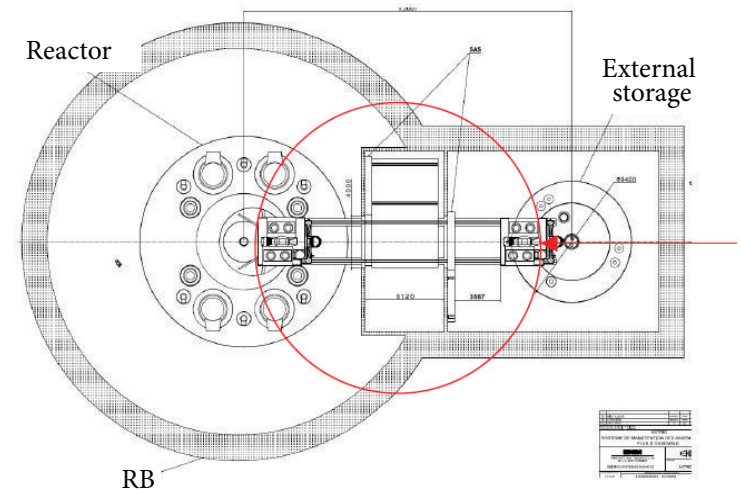

(a)

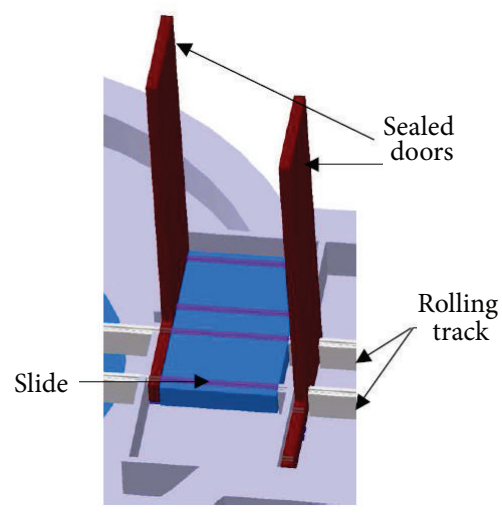

(b)

FIGURE 18: Cross-building airlock.

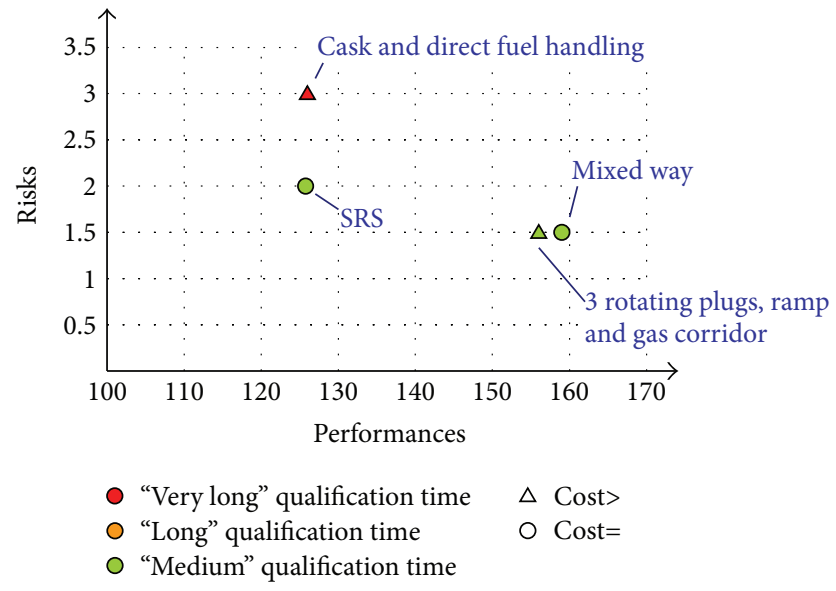

FIGURE 19: Graphical representation of the assessment of solutions.

increased primary vessel diameter, and the extra cost related to complex mechanisms integrated into the cask.

Safety Review. This solution is positive as regards the cooling of the assembly when transferred by the cask but negative due to the complexity of mechanism incorporated into the cask and by the cask being rotated above the reactor core. Therefore, the solution does not seem favourable.

ISIR Review. The absence of any mechanism in the reactor vessel makes these operations easier. The solution is therefore positive.

Operability Review. The solution is better than the SRS due to two casks working in parallel.

Design Complexity Review. The mechanism incorporated into the cask is complex compared to SRS. All the technical issues are focused into one single component: the cask, which therefore accumulates all technological constraints.

Extrapolation to a Power Reactor. The system can be fully extrapolated.

\section{Options Review}

7.1. Advantages/Drawbacks and Difficult Points. The advantages and drawbacks of each solution are listed in Table 1. Major difficulties are marked in bold.

\subsection{Quantification and Comparison of Each Option}

Criteria Grid. Based on the technical elements above, criteria have been generated in order to evaluate and to determine the maturity level of the solutions. A total number of 70 criteria have been analysed to compare each solution in terms of performance and complexity of the design, cost, and availability in-service inspection, risk, and safety. Table 2 is providing a summary of the analysis carried out that focused on cost and risk aspects.

A graphical representation in Figure 19 presents each solution regarding the others.

In Figure 19 four parameters are set: performances ( $x$-axis), risks assessment ( $y$-axis), qualification time (colour from red to green), and investment cost compared to the SRS option (higher $=$ triangle or equivalent $=$ circle). This view simply reveals that the "cask and direct fuel handling" system provides no improvement regarding the SRS option (and safety aspects are very low). The two solutions with gas corridor provide significant improvement in terms of performances with a good confidence in the qualification time. The radar diagram plotted for each solution and based on five major criteria is definitely helping for final decision (Figure 20). 

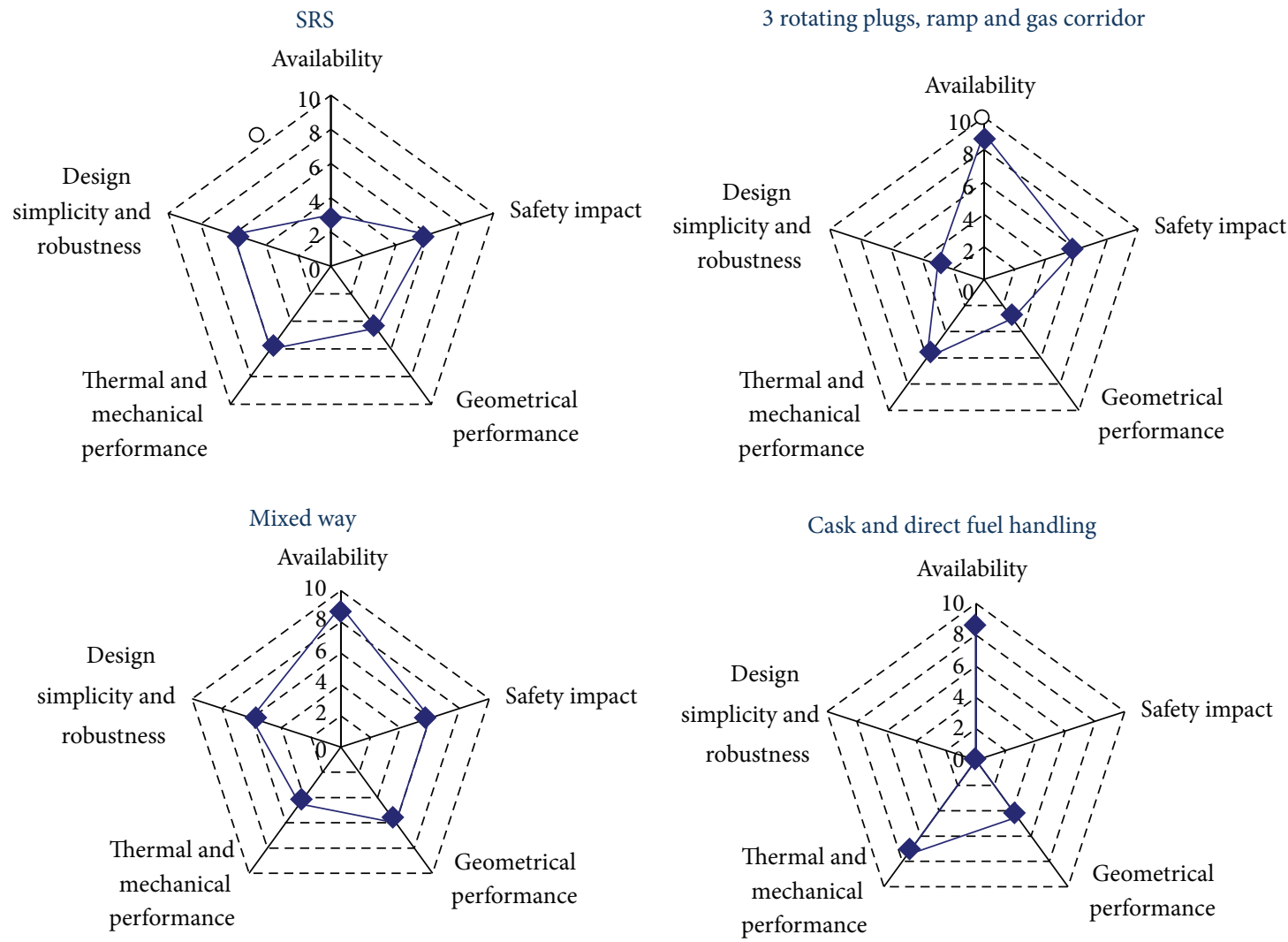

Figure 20: Performance of the options (“radar” diagram).

TABLE 1: Advantages and drawbacks of the reference solution plus the innovative solution investigated.

\begin{tabular}{lc}
\hline Advantages & Drawbacks \\
\hline & SRS option \\
(i) System's simplicity & (i) Fuel handling transfer time \\
(ii) Light impact on the reactor geometry & (ii) Fuel handling cask dimensions (from a thermal and \\
(iii) Easy extrapolability to future reactor commercial & mechanical point of view) \\
& (iii) Large masses being transferred
\end{tabular}

Mixed way-ramp and transfer lock and gas corridor

(i) Limited reactor containment openings

(ii) Low weight in transfer

(iii) Fuel handling duration (i) Thermal features of the corridor

(ii) Components operating under sodium aerosols ambiance

(iii) Sodium drips management

Three rotating plugs, ramp and gas corridor

(i) Improved fuel handling duration

(ii) Reduced weight transferred

(iii) No takeover position

\section{(i) Combination of three rotating plugs and connection} management

(ii) Heavy cask in rotation on rotating plugs

(iii) Increased vessel diameter

(iv) Thermal dimensioning of the gas corridor and quantity to inert

Cask and direct fuel handling

(i) Complex direct extraction system

(i) No mechanism inside the primary vessel

(ii) Limited reactor containment openings

(ii) Increase of the vessel diameter

(iii) Improvement in the fuel handling time is obtained thanks to a complicated system of two cask parallel transfers

(iv) Large reactor containment opening 
TABLE 2: Cost and risks aspects of the three FHR compared to the SRS option.

\begin{tabular}{lcccc}
\hline Criterion proposed & SRS & Mixed way & $\begin{array}{c}3 \text { rotating plugs, } \\
\text { ramp and gas } \\
\text { corridor }\end{array}$ & $\begin{array}{c}\text { Cask and direct } \\
\text { fuel handling }\end{array}$ \\
\hline Manufacturing cost analysis & $=$ & - & $>$ & $>$ \\
Development and qualification & Medium & Medium & Medium & Very long \\
Technical rest (of not achieving performance) & Medium & Medium & Medium & high \\
Safety risks & Medium & low & low & high \\
& 2 & 1,5 & 1,5 & 3 \\
\hline
\end{tabular}

Thus, based on the analysis above, the following orientations are recommended.

(i) Studies on the mixed way-ramp and transfer lock and gas corridor-are pursued due to advantages of this solution, in order to go in detail in its potentiality, especially in the improvement of the load factor and its simplicity in designing the cask.

(ii) The option on the three rotating plugs, ramp and gas corridor is not retained due to technological breakthrough.

(iii) The option on cask and direct fuel handling provides technological difficulties and is not retained.

\section{Conclusions}

This study has revealed that the corridor option has to be reconsidered even if the SRS remains the reference option for ASTRID reactor. Advantages of this mixed way option such as load factor improvement and design simplicity have to be confirmed. The study of a complete fuel handling route (from the primary vessel until the EVST) is interesting in the way that it helps providing new ideas (e.g. the gas corridor or the cross-building airlock). Some specific innovative aspects can therefore be pointed out even if the whole FHR appears to be too challenging for a SFR reactor. Nonetheless the SRS version also involves technological issues, especially the sodium pot cask which has to transfer an assembly at a high residual power (around $40 \mathrm{~kW}$ ). As the continuation of this study, effort will be put on the mixed way-ramp and transfer lock and gas corridor. In parallel the thermal and mechanical aspects of the sodium pot cask have to be performed.

\section{Nomenclature}

ACS: $\quad$ Above Core Structure

ASTRID: Advanced Sodium Technological Reactor for Industrial Demonstration

BOP: $\quad$ Balance of plant

CCS: $\quad$ Core Cover Structure

DHR: Decay heat removal

ECS: Energy conversion system

EVST: External vessel storage tank

FHS: Fuel handling system

FHR: $\quad$ Fuel handling route
lp170ptIHX: Intermediate heat exchanger

HTMP: Horizontal transfer mechanism pot

ISIR: In-service inspection and repair

LRP: $\quad$ Large rotating plug

R\&D: $\quad$ Research and development

RP: $\quad$ Rotating plug

SCO: $\quad$ Specific component optimization

SFR: $\quad$ Sodium-cooled fast reactor

SRP: $\quad$ Small rotating plug

SRS: $\quad$ Starting reference solution.

\section{Conflict of Interests}

The authors declare that there is no conflict of interests regarding the publication of this paper.

\section{Acknowledgments}

The authors would like to thank Bertin/CNIM and COMEX Nucléaire engineering companies for their works and contribution in bringing alternative and innovative solutions to systems which were so far from their current works. In particular the authors provide special thanks to MM. G. Rainaud and D. Dumont for their works on the cask and direct fuel route and MM. D. Roulet and M. Macia and their engineering team for their work on the three rotating plugs, ramp and gas corridor route. M. Saez (CEA) kindly accepted to read this text at all the steps and made important comments, observations, and suggestions now fully integrated in the present version.

\section{References}

[1] F. Gauché and J. Rouault, "French SFR R\&D program and design activities for SFR prototype ASTRID," Energy Procedia, vol. 7, pp. 314-316, 2011.

[2] P. Le Coz, J. F. Sauvage, and J. P. Serpantié, "Sodium-cooled fast reactors: the ASTRID plant project," in Proceedings of the International Conference of Asian Political Parties (ICAPP '11), Nice, France, May 2011.

[3] M. Saez, S. Menou, and B. Uzu, "The pre-conceptual design of the nuclear island of ASTRID," in Proceedings of the International Conference of Asian Political Parties (ICAPP '12), p. 12070, Chicago, Ill, USA, June 2012.

[4] G. Rodriguez, M. Saez, M. Levy et al., "Review and innovative technologies on fuel handling system for sodium fast reactors," 
in Proceedings of the 7th International Conference on Advanced Nuclear Fuel Cycles and Systems (GLOBAL '07), Boise, Idaho, September 2007.

[5] Y. Chikazawa, M. Farmer, and C. Grandy, "Technology gap analysis on sodium-cooled reactor fuel-handling system supporting advanced burner reactor development," Nuclear Technology, vol. 165, no. 3, pp. 270-292, 2009.

[6] G. Prèle, G. Rodriguez, E. Sanseigne, M. Chassignet, and C. Majot, "Some experimental feedback of the fuel handling system of French sodium fast reactors regarding specific operations: cleaning, refuelling, final defueling," in IAEA Technical Meeting on Fuel Handling Systems of Sodium Cooled Fast Reactors, pp. 24-27, IGCAR, Kalpakkam, Tamil Nadu, India, November 2008.

[7] G. Prèle, C. Latgé, R. Dupraz, and J. P. Dirat, "Feedback experience from sodium technology," Revue Générale Nucléaire RGN no. 1, Janvier-Février 2009.

[8] G. Srinivasan, K. V. Suresh Kumar, B. Rajendran, and P. V. Ramalingam, "The fast breeder test reactor-design and operating experiences," Nuclear Engineering and Design, vol. 236, no. 7-8, pp. 796-811, 2006.

[9] K. V. S. Kumar, A. Babu, B. Anandapadmanaban, and G. Srinivasan, "Twenty five years of operating experience with the fast breeder test reactor," Energy Procedia, vol. 7, pp. 323332, 2011.

[10] P. Chellapandi, P. Puthiyavinayagam, V. Balasubramaniyan et al., "Design concepts for reactor assembly components of 500 MWe future SFRs," Nuclear Engineering and Design, vol. 240, no. 10, pp. 2948-2956, 2010.

[11] A. Katoh, Y. Chikazawa, H. Obata, and S. Kotake, "Development of advanced fuel handling machine for JSFR," Journal of Nuclear Science and Technology, vol. 47, no. 7, pp. 642-651, 2010.

[12] D. Cacuci, "Handbook of nuclear engineering," in Sodium Fast Reactor Design: Fuels, Neutronics, Thermal-Hydraulics, Structural Mechanics and Safety, chapter 21, pp. 2321-2711, Springer, New York, NY, USA, 2010.

[13] M. Chassignet, S. Dumas, C. Penigot et al., "Challenges and innovative technologies on fuel handling systems for future sodium-cooled fast reactors," Journal of Nuclear Science and Technology, vol. 48, no. 4, pp. 662-668, 2011.

[14] J. C. Lefèvre, C. H. Mitchell, and G. Hubert, "European fast reactor design," Nuclear Engineering and Design, vol. 162, pp. 133-143, 1994.

[15] F. Dechelette, S. Christin, E. Sanseigne et al., "Study and evaluation of innovative fuel handling systems for SFRs-part 2 : single component optimization," IAEA-CN-199-123, FR13, Paris, France, March 2013.

[16] F. Morin and F. Dechelette, “Transfert innovant d'assemblages combustibles pour un RNRNA de la cuve réacteur vers un stockage externe déporté," French Patent HD 13521, June 2012.

[17] IAEA, "Fast Reactor Database," IAEA TECDOC no. 1531, 2006.

[18] K. J. Henry, “Technical description of PFR," Nuclear Engineering International, vol. 16, no. 183, pp. 632-636, 1971.

[19] C. R. Hunt, P. L. Riley, and N. Campbell, "Fuel handling and other In-reactor mechanisms in PFR," in Proceedings of the International Conference Fast Reactor Power Stations, pp. 11-14, London, UK, March 1974.

[20] D. B. Melhuish, "Engineering improvements to PFR," Nuclear Energy, vol. 31, no. 3, p. 193, 1992. 


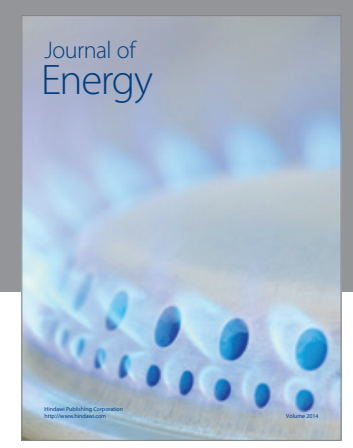

Journal of

Industrial Engineering
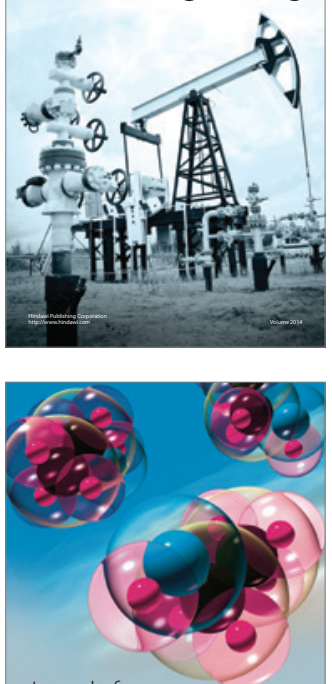

Fuels
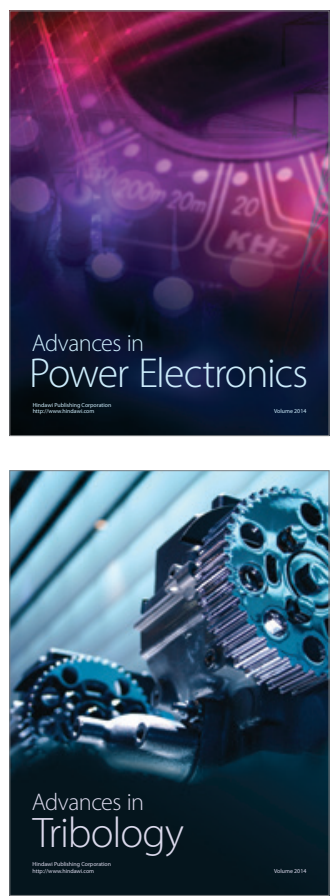

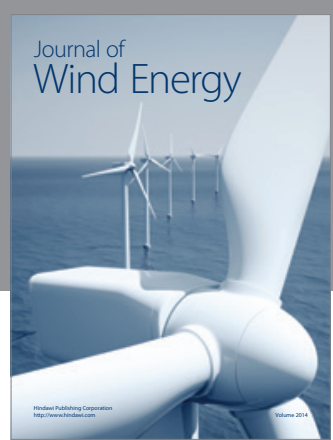

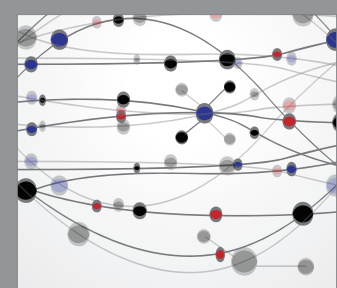

The Scientific World Journal

Submit your manuscripts at http://www.hindawi.com

Journal of

Structures
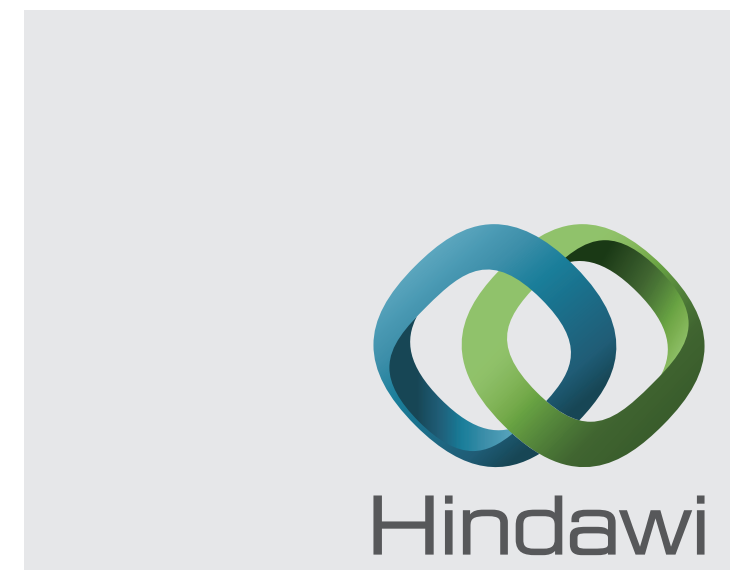

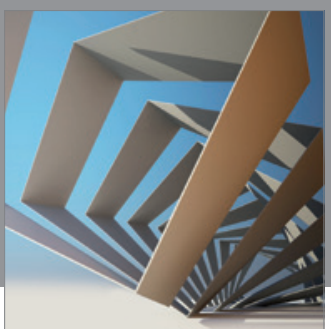

Rotating

Machinery
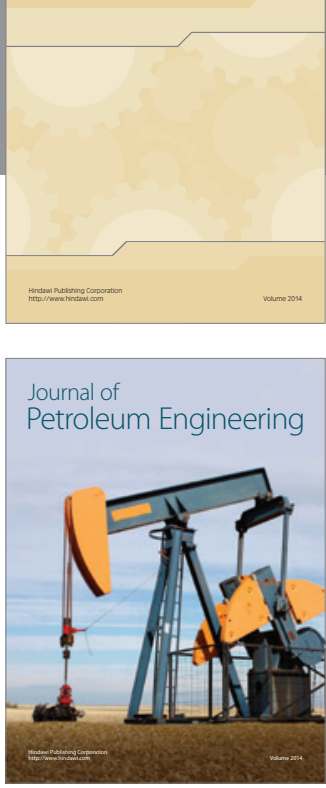

Journal of

Solar Energy
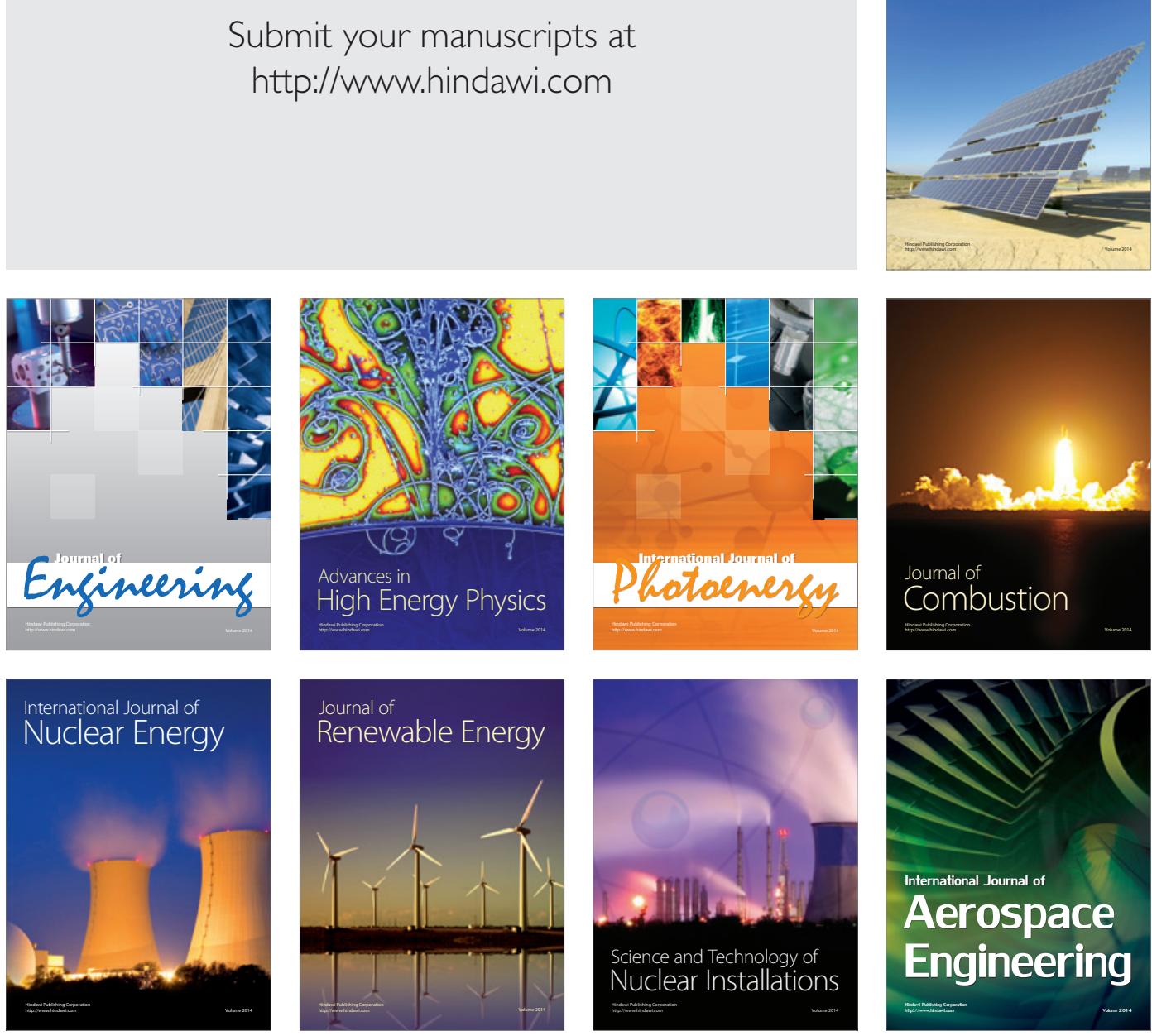